\title{
Torsade de pointes associated with chloroquine, hydroxychloroquine, and azithromycin: a retrospective analysis of individual case safety reports from VigiBase
}

\author{
Diego Macías Saint-Gerons $^{1}$ (1) $\cdot$ Rafael Tabarés-Seisdedos $^{1}$
}

Received: 12 June 2020 / Accepted: 3 April 2021 / Published online: 3 May 2021

○ The Author(s), under exclusive licence to Springer-Verlag GmbH Germany, part of Springer Nature 2021

\begin{abstract}
Purpose To analyze the cases of torsade de pointes (TdP) and related symptoms reported in association with chloroquine (CQ), hydroxychloroquine (HCQ), and azithromycin (AZT) to the World Health Organization (WHO) global database of individual case safety reports (ICSRs) for drug monitoring (VigiBase) using qualitative and quantitative pharmacovigilance approaches.

Methods The main characteristics of the ICSRs reporting TdP with CQ, HCQ, and AZT have been summarized. Co-reported drugs with risk to cause QT prolongation have been described. Reporting odds ratios (RORs) as a measure of disproportionality for reported TdP and individual drugs have been calculated.

Results One hundred seventy ICSRs reporting TdP in association with the drugs of interest were identified (CQ: 11, HCQ: 31, CQ+HCQ: 1, HCQ + AZT: 27, AZT: 100). From these, 41 (24.3\%) were received during the pandemic period (December 2019 to February 2021). The median age of the patients was 63, 53, and 63 years old for CQ, HCQ, and AZT, respectively. Reports included concomitant use of other QT-prolonging drugs (CQ 25.0\%, HCQ 71.2\%, AZT 64.6\%). A proportion of the cases were fatal (CQ 25.0\%, HCQ 8.6\%, AZT 16.1\%). Increased disproportionality has been found for the individual drugs and TdP: CQ (ROR: 7.41, 95\% confidence interval (CI): 3.82, 12.96), HCQ (ROR: 8.49, 95\% CI: 6.57, 10.98), azithromycin (ROR: $8.06,95 \%$ CI: 6.76, 9.61). Disproportionality was also found for other related symptoms, Standardized MedDRA Query for torsade de pointes/QT prolongation (narrow): CQ (ROR: 11.95, 95\% CI: 10.04-14.22); HCQ (ROR: 20.43, 95\% CI: 19.13, 21.83), AZT (ROR: 7.78, 95\% CI: 7.26, 8.34).

Conclusions The prescription of CQ, HCQ, and AZT should be restricted to therapeutic indications with established positive benefit/risk profile. Doctors and patients should be aware of this potential adverse reaction especially when several risk factors are present.
\end{abstract}

Keywords Torsade de pointes $\cdot$ Long QT syndrome $\cdot$ Chloroquine $\cdot$ Hydroxychloroquine $\cdot$ Azithromycin

\section{Introduction}

Since WHO declared international public health emergency, several drugs have been repurposed in an attempt to treat or prevent COVID-19. At the beginning of this global health threat, the Food and Drug Administration (FDA) repurposed chloroquine (CQ) and hydroxychloroquine (HCQ) for the treatment of COVID-19, revoking its emergency

Diego Macías Saint-Gerons

dmacias.sg@gmail.com

1 Department of Medicine, University of Valencia, INCLIVA Health Research Institute and CIBERSAM, Valencia, Spain use authorization several months later [1]. Similarly, the randomized global platform trial for COVID-19 treatments led by the World Health Organization [2] enrolled initially patients taking these drugs, but the trial's HCQ arm was later discontinued. Moreover, combined therapy with azithromycin is also used after one controversial trial suggested a potential benefit $[3,4]$. The urge to find therapeutic solutions has prompted to heterogeneous veering decisions including conditional authorizations, emergency authorizations, or recommendation for drug utilization in compassionate use or off-label use [5].

Despite these drugs have been in the market for decades, the prospect of an increased utilization during the pandemic 
has prompted the interest about the safety of these medicines in a context of uncertain effectiveness [6].

CQ, HCQ, and AZT have been associated with heart rhythm disturbances including torsade de pointes (TdP), a polymorphic ventricular tachycardia often preceded by a prolonged QT interval that can led to life-threatening arrhythmia and sudden cardiac death [7]. Cardiotoxic effects associated with these drugs are considered rare to occur at therapeutic doses but several cases have been reported $[8,9]$.

The aim of the present study was to analyze the reported cases of TdP with chloroquine, hydroxychloroquine, and azithromycin to the World Health Organization (WHO) global database of individual case safety reports (ICSRs) using quantitative and qualitative pharmacovigilance approaches.

\section{Methods}

We searched in World Health Organization (WHO) global database of individual case safety reports (VigiBase) for ICSRs from inception on Nov. 14, 1967, and February 10, 2021. VigiBase is maintained and developed on behalf of WHO by the Uppsala Monitoring Centre (UMC), situated in Uppsala, Sweden, and includes more than 21 million ICSRs (https://www.who-umc.org/). The ICSRs were accessed using the VigiLyze tool. A de-duplicated dataset version of VigiBase was used (database version April 12, 2020).

We searched for the following preferred terms (PTs) of the Medical Dictionary for Regulatory Activities (MedDRA version 22.1; http://www.meddra.org/): "torsade de pointes", "electrocardiogram QT-prolonged", "ventricular tachycardia", "Long QT syndrome" reported for chloroquine, hydroxychloroquine, and azithromycin. We also searched for ICSRs for the Standardized MedDRA Query (SMQ) for "torsade de pointes/QT-prolongation (Broad and Narrow)". SMQs are groupings of PTs related to a defined medical condition that is investigated. The "narrow" scope yields "specificity," while the "broad" search yields "sensitivity." A "broad" scope includes both the "narrow" terms and the additional "broad" terms, which are often of a less-specific nature [10]. PTs included in the SMQ groups are available in table S1 (Supplementary Appendix).

The main characteristics of the ICSRs were described including reporting source, patient age, gender, drug indication, and outcome (recovery, fatal). When available, daily doses were calculated from the information on the prescribed dose and the posology indicated the ICSRs. The induction period was calculated as the time between the start of the drug treatment and clinical diagnosis of TdP in the ICSRs. Co-reported drugs with risk to cause QT prolongation and TdP according to the Arizona Center for Education \& Research on Therapeutics website (https://crediblemeds.org/) were described. The drugs were classified using the AZCERT QT/TdP Risk Categories for Drugs: Known Risk of TdP (category I), Possible Risk of TdP (category II), Conditional Risk of TdP (category III), and Avoid in Congenital Long QT Syndrome (category IV).

Disproportional reporting was investigated through the calculation of the reporting odds ratio with their $95 \%$ confidence interval using Woolf's method. Results of ROR $>1.0$ indicate a higher than expected reporting rate but do not necessarily means differences on the risk probability for the adverse reaction $[11,12]$. To test the consistency of the disproportionality over time, we calculated the cumulative RORs per year in the period between the years in which TdP was first reported in the database to 2020. All analyses were conducted using Stata version 14 (StataCorp LP, College Station, TX, USA).

\section{Results}

Following our search, 5691 ICSRs of TdP were found. From these, 170 ICSRs reported a drug of interest and TdP: 11 with CQ, 31 with HCQ, 1 with both HCQ and CQ, 27 with azithromycin reported in combination with CQ and HCQ, and 99 with azithromycin. Forty-one ICSRs were received during the pandemic period (December 2019 to February 2021), which represents almost one-quarter of the total ICSRs reported during the last three decades. The number of reports in 2020 (as compared with the preceded year) increased $1400 \%$ for HCQ (from 2 to 30 ), $375 \%$ for AZT (4 to 19), and from 0 to 3 in the case of CQ. The combination $\mathrm{HCQ}+\mathrm{AZT}$ was reported only in the pandemic period but not before.

The median age of the patients was 63, 53, and 63 years old for CQ, HCQ, and AZT, respectively. Most reports of CQ and AZT involved women, $75 \%$ and $49.6 \%$, respectively (with $13.3 \%$ of unknown gender for AZT), whereas the reporting by gender was more balanced for HCQ, 35.6\% were in women and $39.0 \%$ in men, sex was not specified in $15(25.4 \%)$. Reports of CQ originated mainly in Europe (66.7\%), whereas America was the most common origin for HCQ and azithromycin reports, $76.3 \%$ and $77.7 \%$, respectively. The most common therapeutic indications reported for CQ and HCQ were COVID-19 and autoimmune diseases; no reports suggested use in malaria. Four reports involving HCQ were overdoses. On the other hand, azithromycin was mainly use for respiratory infections but COVID-19 represented $17.3 \%$ of the cases. Main characteristics of the ICSRs are described in Table 1.

Three $(25.0 \%)$ and 42 (71.2\%) ICSRs mentioned other QT-prolonging drugs in addition to CQ and HCQ respectively and $82(64.6 \%)$ ICSRs in addition to azithromycin. From these, the median number of co-prescribed 
Fig. 1 Cumulative Reporting Odds Ratio (ROR) values for individual dugs and TdP per year (Fig. 1a CQ; Fig. 1b HCQ; Fig. 1c Azithromycin)

QT-prolonging drugs was 1 drug (1-3) for CQ and HCQ and 1 drug (1-5) for azithromycin. At least one AZCERT category I drug was co-reported in $63(49.6 \%)$ reports with azithromycin, $36(61.0 \%)$ reports with HCQ, and in 3 reports with CQ (25.00\%). From the total five and sixty-four QT-prolonging drugs that were co-reported with CQ and HCQ, respectively, AZT (42.2\%) followed by amiodarone (6.3\%) and propofol (6.3\%) was the most co-prescribed with HCQ. From the 129 co-reported QT-prolonging drugs with azithromycin, HCQ (17.8\%), amiodarone (7.0\%), furosemide $(7.00 \%)$, and salbutamol (5.4\%) were the most frequent drugs (Table S2, Supplementary Appendix.)

A very consistent disproportionality was found for the individual drugs and TdP: CQ (ROR: $7.41,95 \% \mathrm{CI}$ : 3.82-12.96), HCQ (ROR: 8.49, 95\% CI: 6.57-10.98), AZT (ROR: 8.06, 95\% CI: 6.76-9.61). Moreover, disproportionality was also found for all related symptoms: Electrocardiogram QT prolonged (PT), long QT syndrome (PT), ventricular tachycardia (PT), and SMQ torsade de pointes/QT prolongation (narrow) (Table 2). Cumulative RORs since the first TdP reported in VigiBase in 1991 show that azithromycin disproportionality has been statistically significant during all the study period, whereas CQ and HCQ reached the threshold of disproportionality in 2011. The cumulative analysis shows that in 2020 there was an increase in the magnitude of the disproportionality for the three drugs and especially for HCQ (Fig. 1).

\section{Discussion}

Increased disproportionality - a subtype of drug pharmacovigilance signal — was found for the three drugs and TdP and also for "electrocardiogram QT prolonged (PT)" which often precedes the TdP. The electrophysiological mechanism for drug-induced TdP is usually explained by the potential of these drugs to block the potassium channels and inhibit the delayed rectifier potassium current (IKr) leading to early after depolarization and resulting in QT interval prolongation [13]. Drug-induced QT/QTc interval prolongation is not specific but a sensitive surrogate indicator for drug-induced TdP risk and despite its limited specificity, it is considered the best marker available for TdP [8, 14]. The threshold of disproportionality was reached using the "narrow SMQ" approach for torsade de pointes/QT prolongation but only a marginal disproportionality was found when the "broad SMQ" was used. The use of broad SMQ describes conditions other than QT prolongation and TdP and can result in
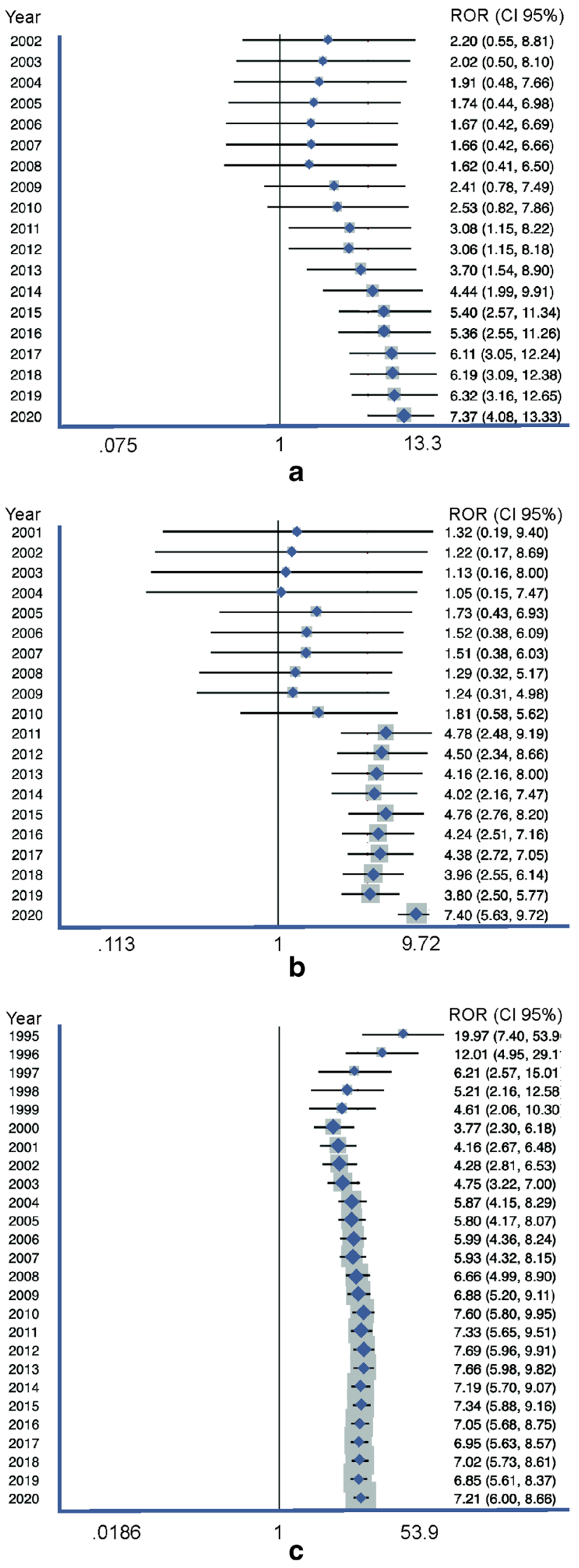


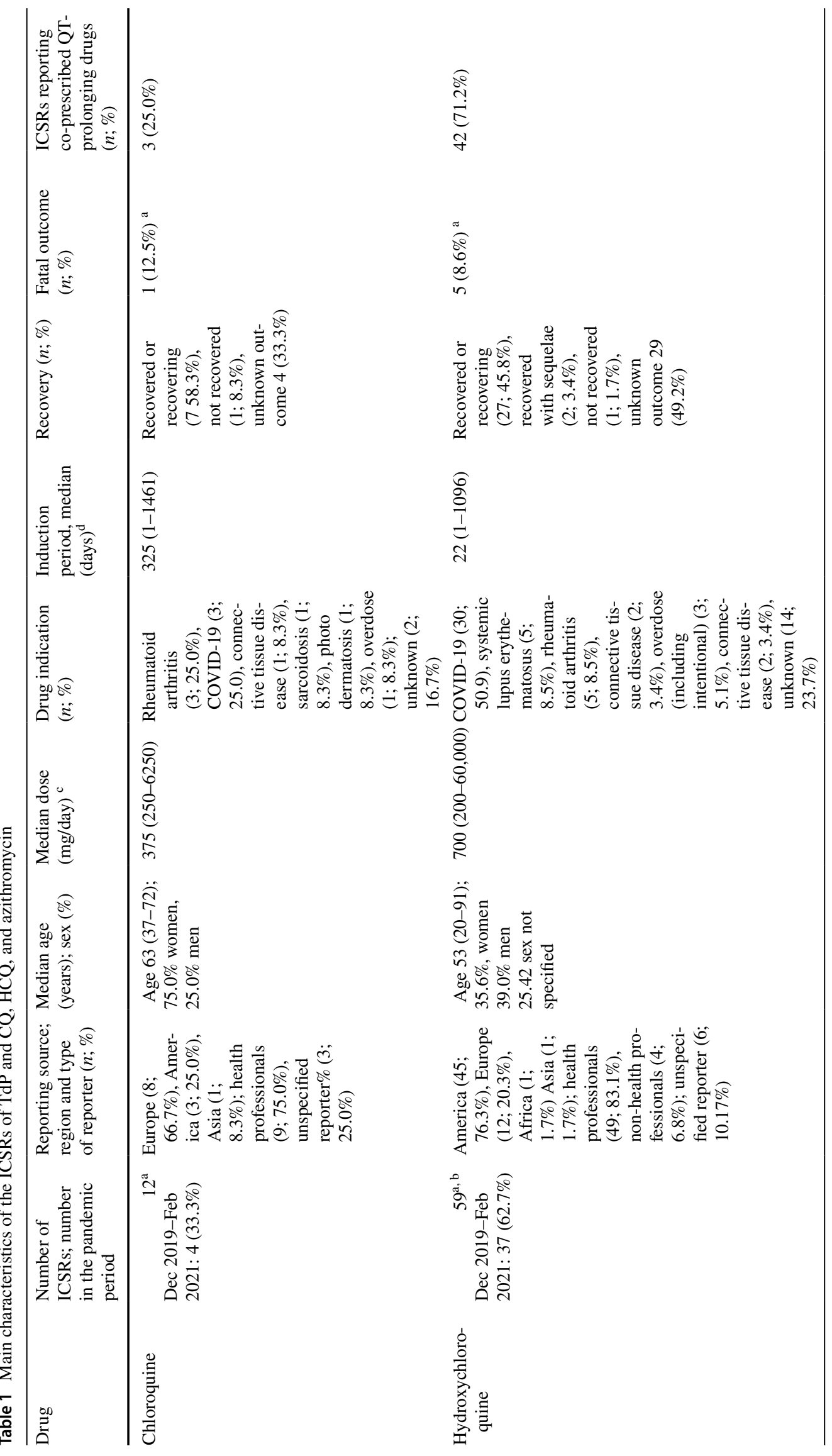




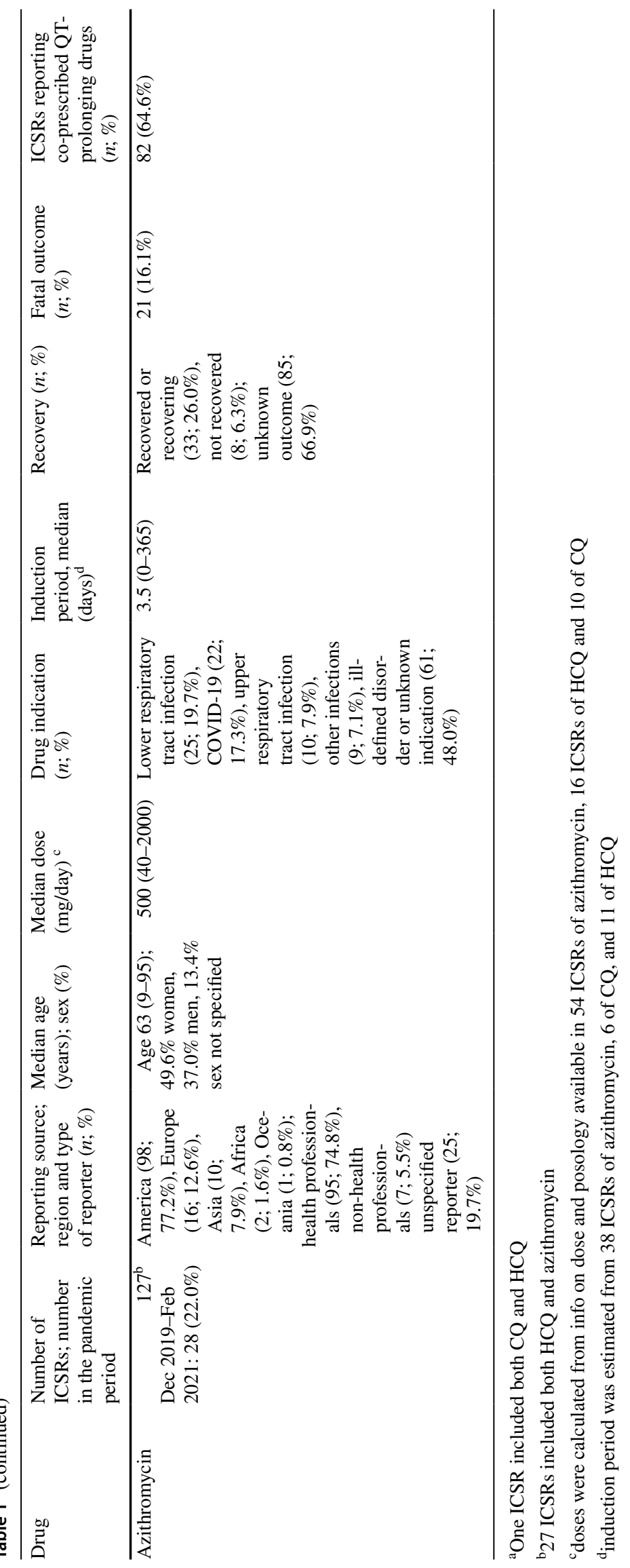


Table 2 Reporting odds ratio (ROR) values for individual drugs and TdP and related symptoms

\begin{tabular}{|c|c|c|c|}
\hline & \multicolumn{2}{|c|}{ Cases/non cases } & \multirow[t]{2}{*}{ ROR $(95 \% \mathrm{CI})$} \\
\hline & Exposed & Non-exposed & \\
\hline \multicolumn{4}{|l|}{ Chloroquine } \\
\hline Torsade de pointes & $12 / 6918$ & $5679 / 24,253,501$ & $7.41(3.82-12.96)$ \\
\hline Electrocardiogram QT prolonged & $111 / 6819$ & $22,216 / 24,236,964$ & $17.41(14.43-21.01)$ \\
\hline Long QT syndrome & $2 / 6928$ & $952 / 24,258,228$ & $7.36(1.84-29.46)$ \\
\hline Ventricular tachycardia & $10 / 6920$ & $13,481 / 24,245,699$ & $2.60(1.40-4.83)$ \\
\hline SMQ torsade de pointes/QT prolongation (broad) & $220 / 6710$ & $356,207 / 23,902,973$ & $2.20(1.92-2.52)$ \\
\hline SMQ torsade de pointes/QT prolongation (narrow) & $130 / 6800$ & $38,752 / 24,220,428$ & $11.95(10.04-14.22)$ \\
\hline \multicolumn{4}{|l|}{ Hydroxychloroquine } \\
\hline Torsade de pointes & $59 / 29,891$ & $5632 / 24,230,528$ & $8.49(6.57-10.98)$ \\
\hline Electrocardiogram QT prolonged & $803 / 29,147$ & $21,524 / 24,214,636$ & $30.99(28.99-33.29)$ \\
\hline Long QT syndrome & $47 / 29,903$ & $907 / 24,235,253$ & $42.00(31.32-56.32)$ \\
\hline Ventricular tachycardia & $77 / 29,873$ & $13,414 / 24,222,746$ & $4.65(3.27-5.82)$ \\
\hline SMQ torsade de pointes/QT prolongation (broad) & $1175 / 28,775$ & $355,252 / 23,880,908$ & $2.74(2.59-2.91)$ \\
\hline SMQ torsade de pointes/QT prolongation (narrow) & $930 / 29,020$ & $37,952 / 24,198,208$ & $20.43(19.13-21.83)$ \\
\hline \multicolumn{4}{|l|}{ Azithromycin } \\
\hline Torsade de pointes & $127 / 68,496$ & $5564 / 24,191,923$ & $8.06(6.76-9.61)$ \\
\hline Electrocardiogram QT prolonged & $613 / 68,010$ & $21,714 / 24,175,773$ & $10.04(9.26-10.88)$ \\
\hline Long QT syndrome & $47 / 68,576$ & $907 / 24,196,580$ & $18.28(13.64-24.52)$ \\
\hline Ventricular tachycardia & $131 / 68,492$ & $13,360 / 24,184,127$ & $3.46(2.91-4.11)$ \\
\hline SMQ torsade de pointes/QT prolongation (broad) & $1452 / 67,171$ & $354,975 / 23,842,512$ & $1.45(1.38-1.53)$ \\
\hline SMQ torsade de pointes/QT prolongation (narrow) & $831 / 67,792$ & $38,051 / 24,159,436$ & $7.78(7.26-8.34)$ \\
\hline
\end{tabular}

$S M Q$ Standardized MedDRA Query

a decreased specificity and a weaker (diluted) signal [10, 15]. Disproportionality was observed for TdP and azithromycin in a previous study performed in the US FDA AERS database [16]. Several individually reported cases of TdP included in this study have been described in detail elsewhere [17-35].

In our study, most cases reporting TdP were in females and co-prescribing with drugs that can prolong the QT was common. Consistently, the effects of drugs in the QT interval have been described to be greater in women [36] and have been considered a risk factor for HCQ-induced TdP $[37,38]$. Moreover, concomitant medication known to prolong the QT may have a synergistic effect in QT prolongation risk. However, co-administration of drugs categorized as "Known Risk of TdP" by the AZCERT (category I) can play a major role in arrhythmia risks beyond the absolute number of co-prescribed drugs [39]. In our study, almost one-third of the reports of HCQ and azithromycin, and a quarter of the reports of CQ have at least one co-reported drug of category I. Congenital hereditary long QT syndrome is another important risk factor that was identified in one report with CQ. Moreover, inherited arrhythmia syndromes occur in younger patients (children or adolescents) [40]. Four reports with azithromycin were in younger than 18 years old.
HCQ and CQ are 4-amino-quinolines antimalarial drugs structurally related to quinidine, an anti-arrhythmic drug with known QT-prolonging effects and that has been also associated with QT-related malignant arrhythmias [40]. Hydroxychloroquine sulfate is the hydroxylated analog of chloroquine [41]. After World War II, these drugs were repurposed as antirheumatic drugs [42]. Drug repositioning (also known as drug repurposing or drug reprofiling) is a process of developing new indications for existing drugs [43]. Despite CQ being the most widely used antimalarial drug in history [9], in our study, reports of TdP and HCQ or CQ mostly originated in Europe and North America for the treatment of autoimmune diseases and for the treatment of COVID-19 from 2020 onwards. Several reasons could be hypothesized to explain the absence of reports for the treatment (or prophylaxis) of malaria. First, the patients treated for malaria are probably younger, healthier, and treated with shorter treatment regimens than the chronic rheumatic patients in which the longer treatments and the autoimmune disease itself are risk factors [37, 44]. Second, the malaria-endemic areas are located in countries in which the pharmacovigilance systems are less mature; thus, the ability to detect and report rare events can be also limited [45]. Third, despite the wide use of these drugs in malaria, very few studies have evaluated its potential arrhythmogenic 
cardiotoxicity. A systematic review found only 1076 participants from 17 studies of chloroquine that underwent ECG investigation making unable pooled estimates of QT interval changes in malaria [46]. The narrow therapeutic range of chloroquine led WHO to recommend that its parenteral use should be discontinued in 1984 [9]. CQ and HCQ are extremely toxic in overdose. Several chloroquine-related deaths have been recently reported in Africa and Arizona [6]. In 2018, the American Association of Poison Control Centers registered three fatal cases associated with HCQ [47]. In our study, four reports were overdoses in the context of suicide attempt. Three patients took $800 \mathrm{mg}, 4 \mathrm{~g}$, and $60 \mathrm{~g}$ of HCQ respectively and recovered after intralipid emulsion therapy $[17,21,28]$. A fourth report was a fatal case after an intentional overdose combining $6.2 \mathrm{~g}$ of $\mathrm{CQ}$ and $6 \mathrm{~g}$ of HCQ.

Azithromycin is a semi-synthetic macrolide antibiotic approved in the early 1990s widely used in monotherapy to treat respiratory tract infections, Chlamydia trachomatis infections, and/or male non-gonococcal urethritis [8]. Azithromycin is one of the most consumed antibiotics in the world [48-52] and has also the highest absolute number of reported cases of TdP (and fatal cases) of the three studied drugs. After a cohort study was published in 2012 [53], the FDA released a warning in 2013 regarding the risk of potentially fatal heart rhythms associated with azithromycin [54]. Despite the potential notoriety bias that might have affected the disproportionality, in our study, the cumulative reporting analyses show that disproportionality was already statistically significant 18 years before the FDA safety announcement. Azithromycin-induced QT prolongation has been shown to start within hours to days of initiating therapy [55]. In our study, the median induction period for TdP with azithromycin was 3.5 days, shorter than in the reports of CQ or HCQ. Nevertheless, results should be interpreted with caution since one third of the ICSRs did not mention the induction period. Subsequent observational studies have reported conflicting findings [55, 56]. The absolute risk seems to be small in general population, but it may be increased in subpopulations with risk factors and comorbidity.

The FDA provided initially an emergency use authorization in COVID-19 for HCQ and CQ. The authorization was revoked months later based on FDA's continued review which concluded that these drugs were unlikely to be effective [2]. Moreover, the latest results from randomized clinical trials had not found any efficacy for these drugs in the treatment of COVID-19 [57-59].

Consistently with the wide use of these drugs, we observed an important increase in the absolute number of reports of CQ, HCQ, and AZT reported from 2020 onwards, which represented almost one-quarter of the total ICSRs reported in the last three decades. TdP was also disproportionately reported higher than expected than other adverse reactions. Despite the revocation of emergency use authorization, seven reports with HCQ and one with chloroquine that originated in the USA were reported in 2021, suggesting that these drugs perhaps are still utilized off-label. It is important to note that safety issues can occur after a drug is repositioned especially when the drug became extensively used and the less known (or less frequent) adverse reactions emerge [60]. Not surprisingly, a small trial $(N=35)$ analyzing the effects of the combined therapy with high doses of chloroquine plus ceftriaxone and azithromycin in COVID-19 was prematurely stopped for safety reasons. Seven (25\%) patients receiving $1200 \mathrm{mg}$ daily of CQ presented QTc prolongation and two (7\%) had ventricular tachycardia before death [61].

\section{Limitations}

Our study also has several limitations. Causal associations cannot (and should not) be made using data from spontaneous reports [50]. Reports of TdP lack full details on the diagnosis (i.e., ECG intervals). Data on known risk factors such as congenital QT syndrome can be lacking from the reports. Moreover, the preferred term "torsade de pointes" although very specific can present some degree of misclassification. Therefore, we have included in the analysis SMQs and related symptoms such as ventricular tachycardia and long QT syndrome and found consistent disproportionality. The possibility of duplicate reporting cannot be excluded. Notwithstanding, a de-duplicated dataset version of VigiBase was used. In addition, the TdP reports were manually reviewed for duplicates. Lastly, spontaneous reporting databases can be useful to identify rare adverse reactions. Pharmacovigilance databases share inherent limitations that have been described elsewhere [62].

In conclusion, the retrospective analysis of a large global pharmacovigilance database has allowed us to find disproportionality at individual drug level which otherwise would have been difficult to address using smaller national databases [63]. The prescription of CQ, HCQ, and AZT should be restricted to therapeutic indications with established positive benefit/risk profile. Doctors and patients should be aware of the potential cardiotoxic effect of these drugs [64]. Therefore, the shared decision for prescribing should balance the benefit against the potential risk of arrhythmia especially when several risk factors are present.

Supplementary Information The online version contains supplementary material available at https://doi.org/10.1007/s00228-021-03133-w.

Acknowledgements We thank the Uppsala Monitoring Centre (Uppsala, Sweden) and the Pan American Network of Pharmacovigilance for their valuable support. We also want to thank to José Luis Castro and Analía Porras from the Unit of Medicines and Health Technologies 
of the Pan American Health Organization for their helpful suggestions and comments during preparation of the manuscript.

Author contribution Conceptualization: Diego Macias Saint-Gerons (DMS), Rafael Taberés-Seisdedos (RTS). Methodology: DMS, RTS. Data analysis: DMS. Writing — original draft preparation: DMS, RTS; Writing-reviewing and editing: DMS, RTS.

\section{Declarations}

Conflict of interest The authors declare no competing interests.

\section{References}

1. Peto R, Henao-Restrepo AM, Preziosi MP, Sathiyamoorthy V, Abdool Karim Q, Alejandria MM, et al (2021) Repurposed antiviral drugs for Covid-19 - Interim WHO solidarity trial results. N Engl J Med 384(6):497-511

2. Food and Drug Administration (FDA) (2020) Fact sheet for health care providers emergency use authorization (EUA) of chloroquine phosphate supplied from the strategic national stockpile for treatment of Covid-19 in certain hospitalized patients: https://www. fda.gov/media/136535/download (accessed April, 2020).

3. Gautret P, Lagier JC, Parola P, Hoang VT, Meddeb L, Mailhe M et al (2020) Hydroxychloroquine and azithromycin as a treatment of COVID-19: Results of an open-label non-randomized clinical trial. Int J Antimicrob Agents. 105949.

4. Ferner RE, Aronson JK (2020) Chloroquine and hydroxychloroquine in Covid-19. BMJ 8(369):m1432

5. Singh JA, Ravinetto R (2020) COVID-19 therapeutics: How to sow confusion and break public trust during international public health emergencies. J Pharm Policy Pract 13:47

6. Juurlink DN (2020) Safety considerations with chloroquine, hydroxychloroquine and azithromycin in the management of SARS-CoV-2 infection. CMAJ. 2020 Apr 8. pii: cmaj.200528. https://doi.org/10. 1503/cmaj.200528.

7. Sarganas G, Garbe E, Klimpel A, Hering RC, Bronder E, Haverkamp W et al (2014) Epidemiology of symptomatic druginduced long QT syndrome and torsade de pointes in Germany. Europace 16(1):101-108

8. Lu ZK, Yuan J, Li M, Sutton SS, Rao GA, Jacob S et al (2015) Cardiac risks associated with antibiotics: Azithromycin and levofloxacin. Expert Opin Drug Saf 14(2):295-303

9. White NJ (2007) Cardiotoxicity of antimalarial drugs. Lancet 7:549-558

10. Sasaoka S, Matsui T, Hane Y, Abe J, Ueda N, Motooka Y et al (2016) Time-to-onset analysis of drug-induced long QT syndrome based on a spontaneous reporting system for adverse drug events. PLoS ONE 11(10):e0164309

11. Rothman KJ, Lanes S, Sacks ST (2004) The reporting odds ratio and its advantages over the proportional reporting ratio. Pharmacoepidemiol Drug Saf 13(8):519-523

12. Macías Saint-Gerons D, Castro JL (2019) Amyotrophic lateral sclerosis associated with statins. A disproportionality analysis of the WHO Program for International Drug Monitoring database (VigiBase). Eur J Clin Pharmacol. 75(4):587-589.

13. Cubeddu LX (2016) Drug-induced inhibition and trafficking disruption of ion channels: Pathogenesis of QT abnormalities and drug-induced fatal arrhythmias. Curr Cardiol Rev 12(2):141-154

14. World Health Organization (WHO) (2017) The cardiotoxicity of antimalarials. Malaria Policy Advisory Committee Meeting. 22
Mar, 2017. https://www.who.int/malaria/mpac/mpac-mar2017erg-cardiotoxicity-report-session2.pdf (accessed April, 2020).

15. Schlit AF, Delaunois A, Colomar A, Claudio B, Cariolato L, Boev $\mathrm{R}$ et al (2017) Risk of QT prolongation and torsade de pointes associated with exposure to hydroxyzine: Re-evaluation of an established drug. Pharmacol Res Perspect 5(3):e00309

16. Poluzzi E, Raschi E, Motola D, Moretti U, De Ponti F (2010) Antimicrobials and the risk of torsades de pointes: The contribution from data mining of the US FDA Adverse Event Reporting System. Drug Saf 33(4):303-314

17. Berkel MV, Taylor A (2018) A rare case of hydroxychloroquine toxicity successfully treated with intralipid emulsion. Therapy Critical Care Medicine 46(1):431

18. Cocco G, Jerie P (2015) Torsades de pointes induced by the concomitant use of ivabradine and azithromycin: An unexpected dangerous interaction. Cardiovasc Toxicol 15(1):104-106

19. Cole JB, Stellpflug SJ, Smith SW (2016) Refractory hypotension and "ventricular fibrillation" with large U waves after overdose. JAMA Intern Med 176(7):1007-1009

20. Digby GC, Pérez Riera AR, Barbosa Barros R, Simpson CS, Redfearn DP, Methot M et al (2011) Acquired long QT interval: A case series of multifactorial QT prolongation. Clin Cardiol 34(9):577-582

21. Ndukwu I, Ghahramani M (2017) Hydroxychloroquine overdose presenting as acquired QT interval prolongation and torsade de pointes. J Am Coll Cardiol 69(11):2340

22. Newton-Cheh C, Lin AE, Baggish AL, Wang H (2011) Case records of the Massachusetts General Hospital. Case 11-2011. A 47-year-old man with systemic lupus erythematosus and heart failure. N Engl J Med. 364(15):1450-60.

23. Park M, Nguyen V, Debord J (2016) A twist of fate. J Gen Intern Med 31(2 Suppl. 1):S533-S534

24. Sheehan ET, Frizzell JD, Gabaldon J, West MB (2016) Quinine and the ABCs of long QT: A patient's misfortune with arthritis, (alcoholic) beverages, and cramps. J Gen Intern Med 31(10):1254-1257

25. Stellpflug S, Floan A, Lee S, Cole J (2013) Hydroxychloroquine overdose treated successfully with intravenous fat emulsion. Clin Toxicol 51(7):652

26. Vereckei A, Fazakas A, Balo T, Fekete B, Molnar MJ, Karadi I (2013) Chloroquine cardiotoxicity mimicking connective tissue disease heart involvement. Immunopharmacol Immunotoxicol 35(2):304-306

27. Yazdan-Ashoori P, Digby G, Baranchuka A (2012) Failure to treat torsades de pointes. Cardiol Res 3(1):34-36

28. Murphy LR, Maskell KF, Kmiecik KJ, Shaffer BM (2018) Intravenous lipid emulsion use for severe hydroxychloroquine toxicity. Am J Ther 25(2):e273-e275

29. Mercuro NJ, Yen CF, Shim DJ, Maher TR, McCoy CM, Zimetbaum PJ, et al (2020) Risk of QT interval prolongation associated with use of hydroxychloroquine with or without concomitant azithromycin among hospitalized patients testing positive for coronavirus disease 2019 (COVID-19). JAMA Cardiol 5(9):1036-1041

30. Masson R, Bakhshi H, Haddad TM (2020) Intermittent left bundle branch block and acute heart failure in trastuzumab-induced cardiotoxicity. BMJ Case Rep 13(7):e236009

31. Lee DH, Armanious M, Huang J, Jeong D, Druta M, Fradley MG (2020) Case of pembrolizumab-induced myocarditis presenting as torsades de pointes with safe re-challenge. J Oncol Pharm Pract 26(6): 1544-1548

32. Chorin E, Wadhwani L, Magnani S, Dai M, Shulman E, NadeauRouthier C et al (2020) QT interval prolongation and torsade de pointes in patients with COVID-19 treated with hydroxychloroquine/azithromycin. Heart Rhythm 17(9):1425-1433 
33. Hummel JP, Maraj I, Taoutel R, Chamoun R, Workman VK, Tran $\mathrm{L}$ et al (2020) Temporal trends in arrhythmogenicity related to treatment of COVID-19 infection. Circ Arrhythm Electrophysiol 13(10):e008841

34. Saleh M, Gabriels J, Chang D, Fishbein J, Qiu M, Mountantonakis SE et al (2020) Safely administering potential QTc prolonging therapy across a large health care system in the COVID-19 era. Circ Arrhythm Electrophysiol 13(11):e008937

35. Huang HD, Jneid H, Aziz M, Ravi V, Sharma PS, Larsen T et al (2020) Safety and effectiveness of hydroxychloroquine and azithromycin combination therapy for treatment of hospitalized patients with COVID-19: A propensity-matched study. Cardiol Ther 9(2):523-534

36. McCauley M, Vallabhajosyula S, Darbar D (2016) Proarrhythmic and torsadogenic effects of potassium channel blockers in patients. Card Electrophysiol Clin 8(2):481-493

37. Joyce E, Fabre A, Mahon N (2013) Hydroxychloroquine cardiotoxicity presenting as a rapidly evolving biventricular cardiomyopathy: Key diagnostic features and literature review. Eur Heart J Acute Cardiovasc Care 2(1):77-83

38. Sauer AJ, Newton-Cheh C (2012) Clinical and genetic determinants of torsade de pointes risk. Circulation 125(13):1684-1694

39. Meid AD, Bighelli I, Mächler S, Mikus G, Carrà G, Castellazzi $M$ et al (2017) Combinations of QTc-prolonging drugs: Towards disentangling pharmacokinetic and pharmacodynamic effects in their potentially additive nature. Ther Adv Psychopharmacol 7(12):251-264

40. Wu CI, Postema PG, Arbelo E, Behr ER, Bezzina CR, Napolitano C, et al (2020) SARS-CoV-2, COVID-19 and inherited arrhythmia syndromes. Heart Rhythm. 2020 Mar 31. pii: S1547-5271(20)30285-X. https://doi.org/10.1016/j.hrthm.2020.03.024. [Epub ahead of print].

41. Ponticelli C, Moroni G (2017) Hydroxychloroquine in systemic lupus erythematosus (SLE). Expert Opin Drug Saf 16(3):411-419

42. Ben-Zvi I, Kivity LP, Shoenfeld Y (2012) Hydroxychloroquine: From malaria to autoimmunity. Clin Rev Allergy Immunol 42(2):145-153

43. Macías Saint-Gerons D, Huerta Álvarez C, García Poza P, Montero Corominas D, de la Fuente Honrubia C (2018) Trazodone utilization among the elderly in Spain. A population based study. Rev Psiquiatr Salud Ment. 11(4):208-215.

44. Nord JE, Shah PK, Rinaldi RZ, Weisman MH (2004) Hydroxychloroquine cardiotoxicity in systemic lupus erythematosus: A report of 2 cases and review of the literature. Semin Arthritis Rheum 33(5):336-351

45. Olsson S, Pal SN, Stergachis A, Couper M (2010) Pharmacovigilance activities in 55 low- and middle-income countries: A questionnaire-based analysis. Drug Saf 33(8):689-703

46. Haeusler IL, Chan XHS, Guérin PJ, White NJ (2018) The arrhythmogenic cardiotoxicity of the quinoline and structurally related antimalarial drugs: A systematic review. BMC Med 16(1):200

47. Gummin DD, Mowry JB, Spyker DA, Brooks DE, Beuhler MC, Rivers LJ et al (2019) 2018 annual report of the American Association of Poison Control Centers' National Poison Data System (NPDS): 36th annual report. Clin Toxicol (Phila) 57(12):1220-1413

48. Adriaenssens N, Coenen S, Versporten A, Muller A, Minalu G, Faes C et al (2011) European Surveillance of Antimicrobial Consumption (ESAC): Outpatient macrolide, lincosamide and streptogramin (MLS) use in Europe (1997-2009). J Antimicrob Chemother. 66 Suppl 6:vi37-45.
49. Day MJ, Spiteri G, Jacobsson S, Woodford N, Amato-Gauci AJ, Cole MJ et al (2018) Stably high azithromycin resistance and decreasing ceftriaxone susceptibility in Neisseria gonorrhoeae in 25 European countries, 2016. BMC Infect Dis 18(1):609

50. Goossens H (2009) Antibiotic consumption and link to resistance. Clin Microbiol Infect 15(Suppl 3):12-15

51. Hicks LA, Taylor TH Jr, Hunkler RJ (2013) U.S. outpatient antibiotic prescribing, 2010. N Engl J Med. 368(15):1461-2

52. World Health Organization (WHO) (2018). WHO report on surveillance of antibiotic consumption: 2016-2018 early implementation. Geneva: World Health Organization. https://www.who.int/ medicines/areas/rational_use/oms-amr-amc-report-2016-2018/en/ (accessed April, 2020).

53. Ray WA, Murray KT, Hall K, Arbogast PG, Stein CM (2012) Azithromycin and the risk of cardiovascular death. N Engl J Med 366(20):1881-1890

54. Food and Drug Administration (FDA) (2013) FDA Drug Safety Communication: Azithromycin (Zithromax or Zmax) and the risk of potentially fatal heart rhythms: https://www.fda.gov/drugs/ drug-safety-and-availability/fda-drug-safety-communicationazithromycin-zithromax-or-zmax-and-risk-potentially-fatal-heart (accessed June, 2020).

55. Trac MH, McArthur E, Jandoc R, Dixon SN, Nash DM, Hackam DG et al (2016) Macrolide antibiotics and the risk of ventricular arrhythmia in older adults. CMAJ 188(7):E120-E129

56. Trifirò G, de Ridder M, Sultana J, Oteri A, Rijnbeek P, Pecchioli $S$ et al (2017) Use of azithromycin and risk of ventricular arrhythmia. CMAJ 189(15):E560-E568

57. RECOVERY Collaborative Group (2021) Azithromycin in patients admitted to hospital with COVID-19 (RECOVERY): A randomised, controlled, open-label, platform trial. Lancet 397(10274):605-612

58. Cavalcanti AB, Zampieri FG, Rosa RG, Azevedo LCP, Veiga VC, Avezum A et al (2020) Hydroxychloroquine with or without azithromycin in mild-to-moderate Covid-19. N Engl J Med 383(21):2041-2052

59. WHO Solidarity Trial Consortium. Repurposed antiviral drugs for Covid-19 - Interim WHO solidarity trial results (2021) N Engl J Med. 384(6):497-511

60. Falzon D, Hill G, Pal SN, Suwankesawong W, Jaramillo E (2014) Pharmacovigilance and tuberculosis: Applying the lessons of thioacetazone. Bull World Health Organ 92(12):918-919

61. Borba MGS, Val FFA, Sampaio VS, Alexandre MAA, Melo GC, Brito M, Mourão MPG et al (2020) Effect of high vs low doses of chloroquine diphosphate as adjunctive therapy for patients hospitalized with severe acute respiratory syndrome coronavirus 2 (SARS-CoV-2) infection: A randomized clinical trial. JAMA Netw Open 3(4):e208857

62. Goldman SA (1998) Limitations and strengths of spontaneous reports data. Clin Ther 20 Suppl C:C40-4.

63. Caster O, Aoki Y, Gattepaille LM (2020) Grundmark B (2020) Disproportionality analysis for pharmacovigilance signal detection in small databases or subsets: Recommendations for Limiting False-Positive Associations. Drug Saf 43(5):479-487

64. Sapp JL, Alqarawi W, MacIntyre CJ, Tadros R, Steinberg C, Roberts JD et al (2020) Guidance on minimizing risk of druginduced ventricular arrhythmia during treatment of COVID-19: A statement from the Canadian Heart Rhythm Society. Can J Cardiol. 2020 Apr 8. pii: S0828-282X(20)30325-1. https://doi.org/ 10.1016/j.cjca.2020.04.003. 